\title{
Application of Support Vector Machine on fMRI Data as Biomarkers in Schizophrenia Diagnosis: A Systematic Review
}

\section{Luca Steardo Jr. ${ }^{1 *}$, Elvira Anna Carbone ${ }^{1}$, Renato de Filippis ${ }^{1}$, Claudia Pisanu ${ }^{2}$, Cristina Segura-Garcia ${ }^{3}$, Alessio Squassina ${ }^{2,4}$, Pasquale De Fazio ${ }^{1}$ and Luca Steardo ${ }^{5,6}$}

OPEN ACCESS

Edited by: Katrin H. Preller,

University of Zurich, Switzerland

Reviewed by: Bingsheng Huang, Shenzhen University, China Wenbin Guo,

Second Xiangya Hospital, Central South University, China

${ }^{*}$ Correspondence: Luca Steardo Jr. steardo@unicz.it

Specialty section: This article was submitted to Neuroimaging and Stimulation, a section of the journal

Frontiers in Psychiatry

Received: 31 January 2020 Accepted: 08 June 2020 Published: 23 June 2020

Citation:

Steardo L Jr., Carbone EA, de Filippis $R$, Pisanu $C$, Segura-Garcia C, Squassina A, De Fazio $P$ and Steardo L (2020) Application of Support Vector

Machine on fMRI Data as

Biomarkers in Schizophrenia Diagnosis: A Systematic Review.

Front. Psychiatry 11:588. doi: 10.3389/fpsyt.2020.00588
${ }^{1}$ Department of Health Sciences, School of Medicine and Surgery, University Magna Graecia of Catanzaro, Catanzaro, Italy, 2 Section of Neuroscience and Clinical Pharmacology, Department of Biomedical Sciences, Faculty of Medicine and Surgery, University of Cagliari, Cagliari, Italy, ${ }^{3}$ Department of Medical and Surgical Science, University of Magna Graecia, Catanzaro, Italy, ${ }^{4}$ Department of Psychiatry, Faculty of Medicine, Dalhousie University, Halifax, NS, Canada, ${ }^{5}$ Department of Physiology and Pharmacology, Faculty of Pharmacy and Medicine, Sapienza University of Rome, Rome, Italy, ${ }^{6}$ Department of Psychiatry, Giustino Fortunato University, Benevento, Italy

Non-invasive measurements of brain function and structure as neuroimaging in patients with mental illnesses are useful and powerful tools for studying discriminatory biomarkers. To date, functional MRI (fMRI), structural MRI (SMRI) represent the most used techniques to provide multiple perspectives on brain function, structure, and their connectivity. Recently, there has been rising attention in using machine-learning (ML) techniques, pattern recognition methods, applied to neuroimaging data to characterize diseaserelated alterations in brain structure and function and to identify phenotypes, for example, for translation into clinical and early diagnosis. Our aim was to provide a systematic review according to the PRISMA statement of Support Vector Machine (SVM) techniques in making diagnostic discrimination between SCZ patients from healthy controls using neuroimaging data from functional MRI as input. We included studies using SVM as ML techniques with patients diagnosed with Schizophrenia. From an initial sample of 660 papers, at the end of the screening process, 22 articles were selected, and included in our review. This technique can be a valid, inexpensive, and non-invasive support to recognize and detect patients at an early stage, compared to any currently available assessment or clinical diagnostic methods in order to save crucial time. The higher accuracy of SVM models and the new integrated methods of ML techniques could play a decisive role to detect patients with SCZ or other major psychiatric disorders in the early stages of the disease or to potentially determine their neuroimaging risk factors in the near future. 


\section{INTRODUCTION}

Schizophrenia (SCZ) is a major psychiatric disorder characterized by positive and negative symptoms, associated with cognitive impairment, leading to a worse outcome and a high impact on global functioning (1). The lifetime prevalence is $0.40 \%$ (2), and it has been estimated that approximately 1 in 200 individuals will be diagnosed with SCZ at some point during their lifetime (3). Even if the diagnosis of schizophrenia is made by observation of the clinical features of the disorder according to the Diagnostic and Statistical Manual of Mental Disorders 5 (DSM-5) (4) or on the ICD (5) criteria, evidences on specific biomarkers that can predict or detect the disease accurately at an early stage are still scarce. (6). It is clear that, considering the biological complexity, the attempt to improve insights into the disease processes is difficult: brain neuroanatomy is intrinsically complex and heterogeneous (7). Non-invasive measurements of brain function and structure, as neuroimaging, are useful and powerful tools for studying discriminatory biomarkers $(8,9)$ in patients with mental disorders. In this regard, brain imaging studies have revealed that functional and structural brain connectivity in the default mode network (DMN), salience network (SN) and central executive network (CEN) are consistently altered in schizophrenia (10). To date, functional MRI (fMRI) and structural MRI (sMRI) represent the most used techniques to provide a multiple perspective on brain function, structure, and its connectivity. Large amounts of imaging data from magnetic resonance imaging (MRI) need to be analyzed by computerized methods that are able to process information and determine the probability of diseases with great precision (11). Rising attention has been given to machine-learning (ML) techniques (i.e. pattern recognition methods) applied to neuroimaging data (12) to identify phenotypes to be translated into clinical practice for early diagnosis $(13,14)$. ML techniques applied to fMRI analyze highly complex data sets and assess the importance and interactions between variables, exploring brain functionality and making accurate predictions $(15,16)$. Machine learning stems from the theory that computers can learn to perform specific tasks without being programmed to do so starting from specific input, thanks to the recognition of patterns in the data. Machine learning uses algorithms that learn from data iteratively. For example, it allows computers to find information, even unknown, without being explicitly told where to look for it (17). Among them, the Support Vector Machine (SVM) represents one of the ML techniques that has shown higher accuracy and precision especially in predicting clinical outcome and severity in schizophrenia patients (14). SVM is a supervised learning model with associated learning algorithms that analyzes data used for classification and regression analysis. This technique has yielded good results applied to fMRI in defining a set of features and information from the various regions of the brain allowing to classify healthy controls and patients affected by SCZ with a potential great translational impact (11).

This review aimed to assess the current state of the evidence about the use of SVM techniques in making diagnostic discrimination in SCZ patients from healthy controls (HC) using as input neuroimaging data from fMRI, according to PRISMA guidelines (18).

\section{MATERIALS AND METHODS}

\section{Search Strategy}

Articles published until September $27^{\text {th }}, 2019$ in PubMed, Embase, MEDLINE, PsychINFO, and the Cochrane Library, without language and time limits, were searched by using the following keywords: (Deep Learning OR DL OR Big data OR Artificial Intelligence $O R$ Machine Learning OR Gaussian process $O R$ Regularized logistic OR Linear discriminant analysis $O R$ LDA OR Random forest OR Least Absolute selection shrinkage operator OR elastic net OR LASSO OR RVM OR relevance vector machine OR pattern recognition OR Computational Intelligence OR Machine Intelligence OR support vector OR SVM OR Pattern classification OR Deep learning) AND Schizophrenia AND (fMRI OR magnetic resonance imaging OR MRI OR functional MRI OR functional-MRI OR functional magnetic resonance imaging). All the selected studies were individually reviewed by two researchers. Reference lists from the included articles were screened for additional studies. The eligible publications have been included and cited in this review.

\section{Assessment of Study Quality}

In this systematic review we applied the Jadad rating system (19) to check the methodological quality of included studies. Jadad's process allows to qualify selected studies according to their transparency and reproducibility, with great validity and reliability evidence, through the description of three simple and easy items: randomization methods, the double-blinding procedure, and the patient's withdrawal and dropout reports. Scores range from 0 to 5 points. The cut-off for inclusion in this study was a Jadad score $\geq 3$.

\section{Selection Criteria}

We selected studies applying SVM as ML techniques with patients diagnosed with Schizophrenia according to the DSMIV, DSM-IV TR, DSM-5 or ICD-10 criteria, chronic SCZ or at first episode of schizophrenia (FES) regardless of antipsychotic medications. We excluded studies without a control group and trials including patients affected by general medical conditions, neurological or psychiatric comorbidity, substance abuse or alcohol dependence, traumatic brain injuries with loss of consciousness, and unclear or unverified psychiatric diagnoses according to the DSM or ICD criteria.

\section{Data Collection and Extraction}

Two authors (RdF and EAC) independently screened all the titles and abstracts of the collected articles, and fully read the texts of papers that met the eligibility criteria. In cases of disagreement, a third researcher (LS) supervised and made the final decision. Data from the extracted article included: publication year, sample size, diagnoses, and all statistical data and features (i.e. accuracy, sensitivity, specificity, brain region or networks). 


\section{RESULTS}

Initially, 660 items were identified, of which 384 articles were eliminated because they did not fulfill the inclusion criteria. The abstracts of the remaining 276 articles were reviewed. Overall, 226 out of 276 articles were excluded because they were not trials (i.e. editorials, letters to editors, reviews, meta-analyses, case reports or different interventions). Then, 28 manuscripts out of 50 papers were further excluded because they did not fulfill the inclusion criteria (e.g. unclear or unverified psychiatric diagnoses, studies considering outcome, costs or therapy or not using MRI); the remaining 22 studies (Table 1) were included in this review (Figure 1).

\section{DISCUSSION}

Included studies were very heterogeneous, and the samples vary in size and clinical characteristics (Table 1). Several features from different brain regions were used as inputs for SVM and focused to investigate how the performance of the model in accuracy, precision, sensitivity, and specificity could be affected by these variables. Studies in this review mostly used and evaluated frontal, temporal, and occipital brain regions. ML techniques were able to detect significantly altered activation patterns or brain connectivity differences in SCZ patients compared to HC. Moreover, this happened quickly, effectively, and efficiently, greatly reducing the number of false negatives, as desirable for a good screening test $(42,43)$. SVM has achieved good results in terms of accuracy and precision in identifying patients with SCZ. This technique can improve the clinical and research tasks due to the repetitiveness of the data. Computers learn from previous processing to produce results and make decisions that are reliable and replicable (17). SVM presents pros and cons. Specifically, an important advantage is that SVM is the most used and well-known machine learning tool, and even when other techniques are validated, they are compared with SVM. It achieves high accuracy level (e.g. 99\%) and is the golden standard to develop new techniques. It can be used for both classification and regression purposes; it allows data repeatability; it can be used in different fields of study, and it represents a great option for future studies. However, it is expensive, and its interpretation is not simple as it requires an experienced and dedicated team $(14,44,45)$.

Pläschke et al. used the resting-state Functional Connectivity (FC) to differentiate SCZ patients from matched HC, reaching a remarkable accuracy, equal to $68 \%$. Interestingly, emotional scenes and face processing, empathic processing, and cognitive action control have proven to be the best networks to accurately discriminate patients from HC. Moreover, the age affects network integrity in a more global way so it could be used as a specific flag of functional dysregulation in particular networks affected in SCZ (33). The results of Bae's study reported a decrease in the global and local network connectivity in SCZ patients compared with $\mathrm{HC}$, especially in the superior right temporal region, in the anterior right cingulate cortex, and the inferior left parietal region with an accuracy of $92.1 \%$, sensitivity of $92 \%$, specificity of $92.1 \%$ and precision $94 \%$ (31). One of the largest studies on SCZ (200 patients vs $200 \mathrm{HC)}$ reported a high diagnostic accuracy (84\%) using data from several locations. Otherwise, significantly poorer accuracy was reached with the use of individual sites, showing a lower connectivity in SCZ patients (28). Su et al. recreated the whole brain functional connectivity in SCZ patients (23) vs HC (23) and related the exact spatial location of the activated brain areas to the emerging symptoms. With $>80 \%$ accuracy authors found an increased FC in SCZ patients group (20). It could probably be explained by an altered cerebral connectivity spread throughout the whole brain, with particular aberrations found in many of the main connections. Altered connectivities in both intra- and inter-hemispherical connections were observed by $\mathrm{Li}$ et al. (37), especially in the right hemisphere more than the left hemisphere (temporal, occipital, insula, and limbic regions). Similar data were confirmed in others studies focusing on altered connections (decreased in the basal ganglia, thalamus, lingual gyrus, and cerebellar vermis and increased in medial temporal lobe and posterior cingulate gyri) (39). Koch et al. reached 93\% accuracy in identifying SCZ patients and were also able to predict the severity of the negative symptoms of patients based on ventricular striatal activation patterns (24). The results of these studies corroborate the idea of the occurrence of dysconnectivity in schizophrenic patients and deepen our knowledge on the pathological mechanisms.

Functional network connectivity (FNC) to capture the internetwork connectivity pattern and autoconnectivity to capture the temporal connectivity of each brain network were proposed as features for SVM technique (22). The authors manage to achieve particularly high accuracy values in order to discriminate patients with SCZ from HC thanks to the integration of these features (autoconnectivity + FNC). Indeed, the final diagnostic and classification accuracy settles in 88.21\% (83.7\% for FNC and $80.2 \%$ for autoconnectivity alone), with a sensitivity of $86.7 \%$ ( $81.4 \%$ for FNC and $78.1 \%$ for autoconnectivity alone) and a specificity of $89.5 \%$ (85.9\% for FNC and $82.2 \%$ for autoconnectivity alone). In one of the first studies, the authors were able to analyze the whole functional connectome both in the patient and in the HC groups. They demonstrated many of the main differences, although general and poorly detailed. Indeed, they weighed three series of network-to-network connections (intra-frontoparietal, intracerebellar, frontoparietal default) considered to be of major importance for SCZ psychopathology and clinical manifestation (23). Another paper examined the role of longand short range functional connectivity (lFC) (sFC) in discriminating patients from their own relatives or HC: SCZ group exhibited an spread in $\mathrm{SFC}$ and IFC in the DMN with an adequate level of accuracy, sensitivity, and specificity (94\%, $92 \%$, $96 \%$, respectively) (27). By analyzing the coherence regional homogeneity (Cohe-ReHo) value, Liu et al. demonstrated that it was decreased in several areas, such as the left postcentral gyrus, right precentral gyrus, left superior temporal gyrus, right middle frontal gyrus, left paracentral lobule, right IPL, and 
TABLE 1 | Summary of included studies classifying schizophrenia using SVM.

\begin{tabular}{|c|c|c|c|c|c|c|c|}
\hline Author, year & Sample size & $\begin{array}{c}\text { Best } \\
\text { accuracy }\end{array}$ & $\begin{array}{l}\text { Other } \\
\text { measures } \\
\text { (sensibility, } \\
\text { specificity, } \\
\text { AUC) }\end{array}$ & Data features as input & $\begin{array}{l}\text { Brain regions and networks } \\
\text { involved }\end{array}$ & $\begin{array}{l}\text { Jadad's } \\
\text { score }\end{array}$ & Comments \\
\hline Su et al. (20) & $\begin{array}{l}\mathrm{N}: 64 \\
-32 \mathrm{SCZ} \\
-32 \mathrm{HC}\end{array}$ & $82,8 \%$ & $\begin{array}{l}\text { Sp: } 81,2 \% \\
\text { Sp :84, } 4 \%\end{array}$ & $\begin{array}{l}90 \text { regions ( } 45 \text { for each } \\
\text { hemisphere) and } 26 \text { areas } \\
\text { (nine in each cerebellar } \\
\text { hemisphere and eight in the } \\
\text { vermis) }\end{array}$ & $\begin{array}{l}\text { Default mode network, } \\
\text { cerebellum, visual network, } \\
\text { sensorimotor network, fronto- } \\
\text { parietal network, cingulo- } \\
\text { opercular network }\end{array}$ & 4 & $\begin{array}{l}\text { The trial is confined to } \\
\text { connectivity analyses. }\end{array}$ \\
\hline $\begin{array}{l}\text { Yang et al. } \\
(21)\end{array}$ & $\begin{array}{l}\mathrm{N}: 40 \\
-20 \mathrm{SCZ} \\
-20 \mathrm{HC}\end{array}$ & $\begin{array}{l}\text { Hybrid } \\
\text { ML } \\
87,3 \%\end{array}$ & $\begin{array}{l}\text { Sn: } 85,8 \% \\
\text { Sp: } 88.8 \%\end{array}$ & $\begin{array}{l}150 \text { SNPs from a database } \\
+ \text { auditory stimuli }\end{array}$ & $\begin{array}{l}\text { Cingulate gyrus, post-/pre- } \\
\text { central gyrus, para-central } \\
\text { lobule, precuneus, superior and } \\
\text { inferior parietal lobule. }\end{array}$ & 5 & $\begin{array}{l}\text { Hybrid ML technique using } \\
\text { together } \mathrm{fMRI} \text { and SNP data for } \\
\text { more accuracy. }\end{array}$ \\
\hline $\begin{array}{l}\text { Arbabshirani } \\
\text { et al. }(22)\end{array}$ & $\begin{array}{l}\text { N: } 370 \\
-195 \text { SCZ } \\
-175 \mathrm{HC}\end{array}$ & $88,2 \%$ & $\begin{array}{l}\text { Sn: } 86,7 \% \\
\text { Sp: } 89,5 \%\end{array}$ & $\begin{array}{l}1128 \text { features for each } \\
\text { subject extracted }\end{array}$ & $\begin{array}{l}\text { Control processes, default- } \\
\text { mode, cerebellar networks, and } \\
\text { subcortical, auditory, visual, } \\
\text { somatomotor regions. }\end{array}$ & 5 & $\begin{array}{l}\text { Functional network connectivity } \\
\text { and autoconnectivity improved } \\
\text { significantly classification results. }\end{array}$ \\
\hline $\begin{array}{l}\text { Watanabe } \\
\text { et al. (23) }\end{array}$ & $\begin{array}{l}\text { N: } 91 \\
-54 \mathrm{SCZ} \\
-67 \mathrm{HC}\end{array}$ & $\begin{array}{l}77- \\
88.2 \%\end{array}$ & N.A. & $\begin{array}{l}\text { Authors described a whole } \\
\text { brain resting state } \\
\text { functional connectome. }\end{array}$ & $\begin{array}{l}\text { Lateral prefrontal cortex, intra- } \\
\text { frontoparietal, frontoparietal } \\
\text { default, intracerebellum } \\
\text { networks. }\end{array}$ & 3 & $\begin{array}{l}\text { Authors assessed three sets of } \\
\text { network-to-network } \\
\text { connections as their role in SZ } \\
\text { psychopathology was } \\
\text { considered crucial. }\end{array}$ \\
\hline $\begin{array}{l}\text { Koch et al. } \\
(24)\end{array}$ & $\begin{array}{l}\mathrm{N}: 98 \\
-44 \mathrm{SCZ} \\
-54 \mathrm{HC}\end{array}$ & $\begin{array}{l}69.3- \\
93.2 \%\end{array}$ & $\begin{array}{l}\text { Sn: } 70.5- \\
100 \% \\
\text { Sp: } 40.9- \\
93.2 \%\end{array}$ & $\begin{array}{l}\text { Six fMRI volumes per trial } \\
\text { were acquired, resulting in } \\
\text { a total of } 450 \text { volumes per } \\
\text { run }\end{array}$ & $\begin{array}{l}\text { Nucleus accumbens, amygdala, } \\
\text { insula, thalamus, ventral } \\
\text { striatum, right pallidum, } \\
\text { putamen, right inferior frontal } \\
\text { gyrus, inferior temporal gyrus }\end{array}$ & 5 & $\begin{array}{l}\text { Able to use the ventricular } \\
\text { striatal activation patterns to } \\
\text { predict the severity of the } \\
\text { negative symptoms of patients } \\
\text { enrolled. }\end{array}$ \\
\hline $\begin{array}{l}\text { Chyzhyk } \\
\text { et al. }(25)\end{array}$ & $\begin{array}{l}\mathrm{N}: 147 \\
-72 \mathrm{SCZ} \\
-75 \mathrm{HC}\end{array}$ & $\approx 90 \%$ & N.A. & $\begin{array}{l}\text { rs-fMRI data were collected } \\
\text { with single-shot full } k \text {-space } \\
\text { EPI with ramp sampling } \\
\text { correction using the AC-PC } \\
\text { as a reference. }\end{array}$ & $\begin{array}{l}\text { Inferior temporal gyrus, para- } \\
\text { hippocampal gyrus, planum } \\
\text { polare, thalamus, temporal } \\
\text { fusiform cortex }\end{array}$ & 4 & $\begin{array}{l}\text { Application of SVM and RF } \\
\text { methods to the data extracted } \\
\text { from cross-validation as the } \\
\text { ensembles of ELM }\end{array}$ \\
\hline Liu et al. (26) & $\begin{array}{l}\text { N: } 79 \\
\text { - } 48 \text { Drug- } \\
\text { naïve SCZ } \\
-31 \mathrm{HC}\end{array}$ & $89.9 \%$ & $\begin{array}{l}\text { Sn: } 91.67 \% \\
\text { Sp: } 87.10 \%\end{array}$ & $\begin{array}{l}\text { The fMRI scan lasted for } \\
480 \text { s for every pt included, } \\
\text { and in total } 240 \text { volumes } \\
\text { were obtained. } \\
\text { The first } 10 \text { volumes of } \\
\text { each take-over were } \\
\text { discarded to certain steady } \\
\text { state conditions }\end{array}$ & $\begin{array}{l}\text { Left paracentral lobule, left } \\
\text { postcentral gyrus, left superior } \\
\text { temporal gyrus, right middle } \\
\text { frontal gyrus, bilateral } \\
\text { precuneus, right pre-central } \\
\text { gyrus, right inferior parietal } \\
\text { lobule. }\end{array}$ & 4 & $\begin{array}{l}\text { Authors enrolled only } \\
\text { adolescent onset without } \\
\text { previous medication SCZ } \\
\text { patients }\end{array}$ \\
\hline $\begin{array}{l}\text { Guo et al. } \\
(27)\end{array}$ & $\begin{array}{l}\mathrm{N}: 96 \\
-28 \mathrm{SCZ} \\
-40 \mathrm{HC} \\
-28 \text { relatives }\end{array}$ & $94.6 \%$ & $\begin{array}{l}\text { Sn: } 92.9 \% \\
\text { Sp: } 96.4 \%\end{array}$ & $\begin{array}{l}\text { Long-range and short- } \\
\text { range FCs }\end{array}$ & $\begin{array}{l}\text { Default-mode network, left } \\
\text { fusiform gyrus, cerebellum, } \\
\text { sensorimotor circuits, right } \\
\text { superior parietal lobule }\end{array}$ & 4 & $\begin{array}{l}\text { The SCZ group was } \\
\text { unmedicated and recent onset, } \\
\text { so, results may be confounded } \\
\text { by their acute positive } \\
\text { symptoms }\end{array}$ \\
\hline $\begin{array}{l}\text { Orban et al. } \\
(28)\end{array}$ & $\begin{array}{l}\text { N: } 382 \\
-191 \mathrm{SCZ} \\
-191 \mathrm{HC}\end{array}$ & $84 \%$ & N.A. & $\begin{array}{l}\text { Functional brain } \\
\text { connectomes included a } \\
\text { total of } 2016 \text { functional } \\
\text { connections among } 64 \\
\text { brain parcels }\end{array}$ & Connectivity of the whole brain & 3 & $\begin{array}{l}\text { Brain imaging data derived from } \\
\text { six different and independent } \\
\text { studies and databases. }\end{array}$ \\
\hline $\begin{array}{l}\text { Wang et al. } \\
\text { (29) }\end{array}$ & $\begin{array}{l}\text { N: } 79 \\
-48 \text { AOS } \\
-31 \mathrm{HC}\end{array}$ & $90.1 \%$ & $\begin{array}{l}\text { Sn: } 88.2 \% \\
\text { Sp: } 91.9 \%\end{array}$ & $\begin{array}{l}\text { Authors used brain regions } \\
\text { with significantly different } \\
\text { ReHo values between SCZ } \\
\text { and HC group }\end{array}$ & $\begin{array}{l}\text { Bilateral superior medial pre- } \\
\text { frontal cortex, right inferior } \\
\text { parietal lobule, left paracentral } \\
\text { lobule, left superior temporal } \\
\text { gyrus, right precentral lobule }\end{array}$ & 5 & $\begin{array}{l}\text { Sample sizes in the two groups } \\
\text { were different. }\end{array}$ \\
\hline $\begin{array}{l}\text { Wang et al. } \\
(30)\end{array}$ & $\begin{array}{l}\text { N: } 79 \\
-48 \text { drug- } \\
\text { naïve } \\
-31 \mathrm{HC}\end{array}$ & $92.4 \%$ & $\begin{array}{l}\text { Sn: } 89.6 \% \\
\text { Sp: } 96.8 \%\end{array}$ & $\begin{array}{l}\text { Regional homogeneity } \\
\text { (ReHo), a measurement } \\
\text { that reflects brain local } \\
\text { functional connectivity or } \\
\text { synchronization }\end{array}$ & $\begin{array}{l}\text { Left superior temporal gyrus, } \\
\text { right middle frontal gyrus, right } \\
\text { superior medial prefrontal cortex }\end{array}$ & 4 & $\begin{array}{l}\text { SVM analysis was applied to an } \\
\text { independent database }\end{array}$ \\
\hline $\begin{array}{l}\text { Bae et al. } \\
(31)\end{array}$ & $\begin{array}{l}\mathrm{N}: 75 \\
-21 \mathrm{SCZ} \\
-54 \mathrm{HC}\end{array}$ & $92.1 \%$ & $\begin{array}{l}\text { Sn: } 92.0 \% \\
\text { Sp: } 92.1 \%\end{array}$ & $\begin{array}{l}90 \text { ROls from the image } \\
\text { database. }\end{array}$ & $\begin{array}{l}\text { Anterior right cingulate cortex, } \\
\text { inferior left parietal region, } \\
\text { superior right temporal region }\end{array}$ & 5 & $\begin{array}{l}\text { Likely interference of } \\
\text { pharmacological treatment and } \\
\text { disease phase on the }\end{array}$ \\
\hline
\end{tabular}


TABLE 1 | Continued

\begin{tabular}{|c|c|c|c|c|c|c|c|}
\hline Author, year & Sample size & $\begin{array}{c}\text { Best } \\
\text { accuracy }\end{array}$ & $\begin{array}{l}\text { Other } \\
\text { measures } \\
\text { (sensibility, } \\
\text { specificity, } \\
\text { AUC) }\end{array}$ & Data features as input & $\begin{array}{l}\text { Brain regions and networks } \\
\text { involved }\end{array}$ & $\begin{array}{l}\text { Jadad's } \\
\text { score }\end{array}$ & Comments \\
\hline & & & $\begin{array}{l}\text { Precision: } \\
94 \%\end{array}$ & & & & $\begin{array}{l}\text { investigated functional } \\
\text { connections. Moreover, authors } \\
\text { used only n-back tests without } \\
\text { rs-fMRI }\end{array}$ \\
\hline $\begin{array}{l}\text { Qureshi et al. } \\
\text { (32) }\end{array}$ & $\begin{array}{l}\mathrm{N}: 144 \\
-72 \mathrm{SCZ} \\
-72 \mathrm{HC}\end{array}$ & $99.3 \%$ & $\begin{array}{l}\text { Sn: } 100 \% \\
\text { Sp: } 98,6 \%\end{array}$ & $\begin{array}{l}\text { Mean cortical thickness, } \\
\text { white matter volume, } \\
\text { surface area, volume, } \\
\text { cortical thickness standard } \\
\text { deviation, mean curvature, } \\
\text { subcortical segment } \\
\text { volume, subcortical } \\
\text { intensity, and overall brain } \\
\text { volume and intensity as the } \\
\text { structural features }\end{array}$ & $\begin{array}{l}\text { Surface area, cortical thickness, } \\
\text { global average functional } \\
\text { connectivity, WM/subcortical/ } \\
\text { overall volume, curvature }\end{array}$ & 4 & $\begin{array}{l}\text { Authors developed a specific } \\
\text { ELM in this trial }\end{array}$ \\
\hline $\begin{array}{l}\text { Pläschke } \\
\text { et al. (33) }\end{array}$ & $\begin{array}{l}\mathrm{N}: 170 \\
-86 \mathrm{SCZ} \\
-84 \mathrm{HC}\end{array}$ & $61-72 \%$ & $\begin{array}{l}\text { Sn: } 65-77 \% \\
\text { Sp: } 46-69 \% \\
\text { AUC: } 0.61- \\
0.79\end{array}$ & 12 functional networks. & $\begin{array}{l}\text { Emotion-processing, empathy, } \\
\text { and cognitive action control } \\
\text { networks }\end{array}$ & 3 & $\begin{array}{l}\text { Young-old classification was } \\
\text { grounded on outperformed } \\
\text { clinical classification and all } \\
\text { networks. }\end{array}$ \\
\hline Liu et al. (34) & $\begin{array}{l}\text { N: } 79 \\
-48 \text { Drug- } \\
\text { Naïve FES } \\
-31 \mathrm{HC}\end{array}$ & $94.93 \%$ & $\begin{array}{l}\text { Sn: } 100 \% \\
\text { Sp: } 87.09 \%\end{array}$ & $\begin{array}{l}\text { A total of } 240 \text { volumes } \\
\text { were acquired. } \\
\text { The first } 10 \text { volumes of } \\
\text { each scan were discarded } \\
\text { to certain steady-state } \\
\text { conditions at the beginning } \\
\text { of acquisition. }\end{array}$ & $\begin{array}{l}\text { Superior temporal gyrus, insula, } \\
\text { fusiform gyrus, precentral gyrus, } \\
\text { and precuneus }\end{array}$ & 4 & $\begin{array}{l}\text { Authors assessed also a } \\
\text { neurocognitive test battery } \\
\text { demonstrating neurocognitive } \\
\text { deficits in patients compared to } \\
\text { HC }\end{array}$ \\
\hline $\begin{array}{l}\text { Vacca et al. } \\
(35)\end{array}$ & $\begin{array}{l}\text { N: } 201 \\
-86 \text { SCZ } \\
-115 \mathrm{HC}\end{array}$ & $87,8 \%$ & N.A. & $\begin{array}{l}\text { Battery tests related to } \\
\text { attention, memory, praxic, } \\
\text { visuospatial and executive } \\
\text { functions }\end{array}$ & $\begin{array}{l}\text { Working memory, executive } \\
\text { functions, attention, verbal } \\
\text { fluency, memory }\end{array}$ & 3 & $\begin{array}{l}\text { Data obtained should be } \\
\text { integrated thorough } \\
\text { neuropsychological evaluation } \\
\text { into the more general diagnostic } \\
\text { approach of patients with SCZ }\end{array}$ \\
\hline $\begin{array}{l}\text { Zhuang et al. } \\
\text { (36) }\end{array}$ & $\begin{array}{l}\text { N: } 69 \\
-40 \text { drug } \\
\text { naïve FES } \\
-29 \text { HC }\end{array}$ & $84,29 \%$ & $\begin{array}{l}\text { Sn:92.5 } \\
\text { Sp: } 73.33\end{array}$ & $\begin{array}{l}\text { Structural MRI, diffusion } \\
\text { tensor imaging (DTI) and rs- } \\
\text { fMRI data }\end{array}$ & $\begin{array}{l}\text { Altered morphological } \\
\text { measurements in both gray } \\
\text { matter and white matter, } \\
\text { functional connectivity, and } \\
\text { regional functional activity }\end{array}$ & 5 & $\begin{array}{l}\text { A multimodal classification } \\
\text { method to discriminate FES } \\
\text { schizophrenia patients from HC } \\
\text { by a combined structural MRI, } \\
\text { DTI, and rs-fMRI data }\end{array}$ \\
\hline Li et al. (37) & $\begin{array}{l}\text { N: } 148 \\
-60 \mathrm{SCZ} \\
-71 \mathrm{HC}\end{array}$ & $71,8 \%$ & $\begin{array}{l}\text { Sn: } 70 \\
\text { Sp: } 73,24\end{array}$ & $\begin{array}{l}\text { Aberrant connectivities in } \\
\text { both intra- and inter- } \\
\text { hemispherical connections }\end{array}$ & $\begin{array}{l}\text { Disconnectivities mainly } \\
\text { appeared on temporal and } \\
\text { occipital regions for the within- } \\
\text { large-region connections; } \\
\text { connectivity disruption was } \\
\text { observed on the connections } \\
\text { from temporal region to } \\
\text { occipital, insula and limbic } \\
\text { regions for the between-large- } \\
\text { region connections }\end{array}$ & 4 & $\begin{array}{l}\text { The findings of this study } \\
\text { corroborate previous conclusion } \\
\text { of dysconnectivity in SCZ and } \\
\text { further shed light on distribution } \\
\text { patterns of dysconnectivity, } \\
\text { which deepens the } \\
\text { understanding of its pathological } \\
\text { mechanism. }\end{array}$ \\
\hline $\begin{array}{l}\text { Jing et al. } \\
(38)\end{array}$ & $\begin{array}{l}\text { N: } 153 \\
-60 \text { SCZ } \\
-43 \\
\text { unaffected } \\
\text { FDRs of } \\
\text { patients } \\
-50 \mathrm{HC}\end{array}$ & $83,9 \%$ & $\begin{array}{l}\text { Sn: } 87.5 \% \\
\text { Sp: } 80.0 \% \\
\text { AUC: } 0.914\end{array}$ & Informative FNs & $\begin{array}{l}\text { Cerebellum, default mode } \\
\text { network (DMN), ventral } \\
\text { frontotemporal network, and } \\
\text { posterior DMN with } \\
\text { parahippocampal gyrus }\end{array}$ & 5 & $\begin{array}{l}\text { Pattern classifiers built upon the } \\
\text { informative FNs can serve as } \\
\text { biomarkers for quantifying brain } \\
\text { alterations in SCZ and help to } \\
\text { identify FDRs with FN patterns } \\
\text { and cognitive impairment similar } \\
\text { to those of SCZ patients. }\end{array}$ \\
\hline $\begin{array}{l}\text { Ramkiran } \\
\text { et al. (39) }\end{array}$ & $\begin{array}{l}N: 112 \\
-56 \mathrm{SCZ} \\
-56 \mathrm{HC}\end{array}$ & $69 \%$ & $\begin{array}{l}\text { Sn: } 68 \% \\
\text { Sp: } 72 \%\end{array}$ & Functional connectivity & $\begin{array}{l}\text { The basal ganglia, thalamus, } \\
\text { lingual gyrus, and cerebellar } \\
\text { vermis showed significantly } \\
\text { different, type A (decreased } \\
\text { anticorrelation) connections. The } \\
\text { medial temporal lobe and } \\
\text { posterior cingulate gyri showed }\end{array}$ & 4 & $\begin{array}{l}\text { Different aberrant functional } \\
\text { connectivity in SCZ patients. }\end{array}$ \\
\hline
\end{tabular}


TABLE 1 | Continued

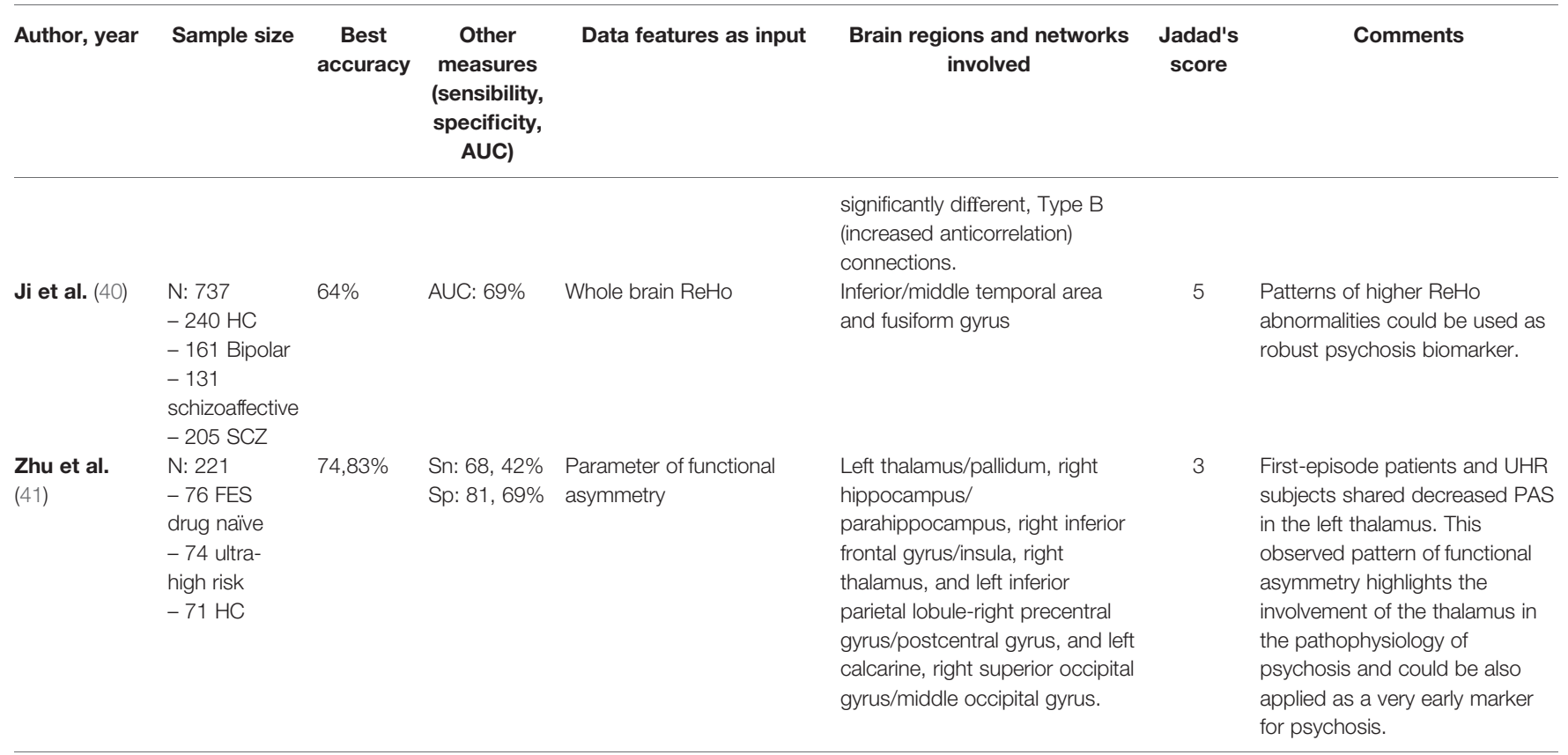

AOS., adolescent onset SCZ; AUC, area under the curve; DMN, default mode network; DTI, diffusion tensor imaging; ELM, extreme learning machine; FC, functional connectivity; FN, functional network; FDR, first-degree relatives; FES, first episode schizophrenia; HC, healthy controls; ML, machine learning; $n$, number; NA, not available; PAS, parameter of asymmetry;

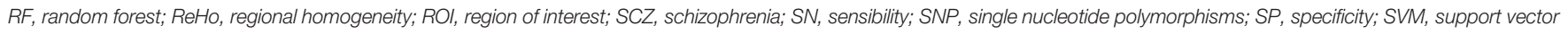
machine; UHR, ultra-high risk.

bilateral praecuneus in 48 SCZ vs $31 \mathrm{HC}$ (26). The Whole brain ReHo measures were used as robust psychosis biomarker: SVM resulted more accurate in identify patterns of higher ReHo abnormalities (inferior/middle temporal area and fusiform gyrus) (40). The integration of the neuropsychological evaluation to detect different aspects related to attention, working memory, praxic, visuospatial, and executive functions was able for the early diagnosis of patients with SCZ (35).

The combination of SVM with other ML techniques can identify anatomic brain areas with major alterations (temporal fusiform cortex, inferior, middle, and medial frontal gyri, inferior temporal gyrus, anterior division of the parahippocampal gyrus, planum polare, cingulate gyrus, superior temporal gyrus, precuneus left, and right thalamus) with an accuracy close to $90 \%(21,25)$. An extreme learning machine (ELM) was developed by Qureshi et colleagues, reaching a maximum accuracy of $99.3 \%$. Main data derived from cortical thickness and surface area, total cerebral volume, and overall volume of cortex features scans. Authors concluded that their ELM technique can be applied to patients offering a solid chance of helping clinicians to make diagnosis of SCZ (32).

Another important field of application of SVM is the evaluation of functional features in first episode schizophrenia (FES). The identification of early-onset schizophrenia remains challenging, and SVM may constitute a promising tool for the early diagnosis for its high accuracy and valuable prognostic implication in FES. Recently, the $\mathrm{sFC}$ and IFC in the whole brain were explored in 48 firstepisode, drug-naïve patients and 31 HC using SVM. Major abnormalities were found in some brain networks (anterior and posterior Default Mode Network and Sensorimotor Network) classifying patients and controls with > 92\% accuracy and high sensitivity and specificity (30). Liu et al. evaluated the alteration in FC in different brain regions in a similar patients' sample and found dysfunctional interhemispheric network within the sensorimotor area among patients with SCZ. It was associated with processing speed deficits, indicating the probable involvement with the neurocognitive alterations of these patients. The application of SVM ML technique analysis reached 100\% sensitivity, 87.09\% specificity, and $94.93 \%$ accuracy (34). Functional alterations could point to a role of DMN and SN in the SCZ psychopathology that is already known in first-psychotic episode patients and SVM seems to be able to discriminate with high accuracy patients from $\mathrm{HC}$ in research context. Wang et al. identify brain peculiarities using ReHo input in SVM analysis through resting state-fMRI (rs-fMRI) in drug-naïve patients and $32 \mathrm{HC}$. ReHo values were significantly amplified in the bilateral superior medial prefrontal cortex, and, otherwise, reduced in the left superior temporal gyrus, right precentral lobule, right inferior parietal lobule, and left paracentral lobule in patient group compared to HC (29). Disrupted functional asymmetry was calculated comparing patients with FES, drug-naive schizophrenia, ultra-high risk (UHR) for psychosis and HC. SVM classification analysis was applied to analyze the data and showed decreased parameter of asymmetry in the left thalamus/pallidum, right hippocampus/parahippocampus, right inferior frontal gyrus/ insula, right thalamus, and left inferior parietal lobule, and increased PAS in the left calcarine, right superior occipital gyrus/middle occipital gyrus, and right precentral gyrus/postcentral gyrus. Firstepisode patients and UHR subjects shared decreased pattern of functional asymmetry in the left thalamus underlining the possible involvement of the thalamus in the pathophysiology of psychosis 


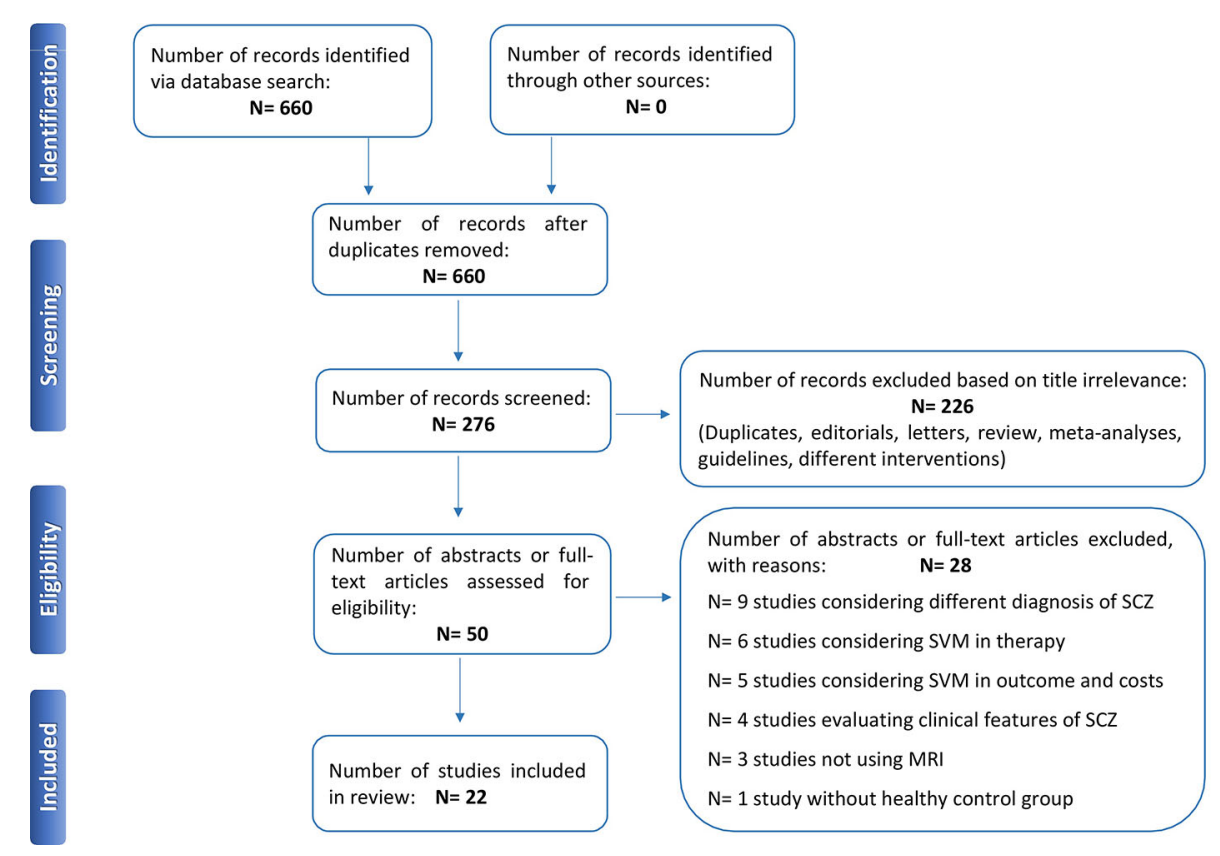

FIGURE 1 | PRISMA flowchart of included studies.

and demonstrating a very early marker for psychosis (41). A multimodal classification method to discriminate FES patients from HC combined structural MRI and rs-fMRI data, and identified functional markers in both gray matter and white matter and altered functional connectivity in DMN and cerebellar connections (36). A recent study identified informative functional networks to distinguish patients from $\mathrm{HC}$ and to classify unaffected first-degree relatives (FDRs) with or without functional networks similar to patients. Four informative functional networks (DMN, ventral frontotemporal network, and posterior DMN with parahippocampal gyrus) resulted implicated in brain alterations. They could be probably used as biomarkers to identify FDRs with FN patterns similar to those of SCZ patients (38). The ability to apply complex mathematical calculations to big data is newly developed, and its use is hopefully growing. Now, theoretically, it is possible to create automatically models for analyzing larger and more complex data and to produce more accurate and repeatable results even on a large scale.

The application of these models would allow clinicians to identify new tasks, not merely diagnostic but also preventive, for major psychiatric disorders such as Schizophrenia.

\section{CONCLUSION}

Approaches of big data, focusing on classification based on huge biological information rather than the single clinical manifestation, have the greatest advantage to move the field forward faster and with more evidence than before. The application of ML techniques in psychiatry as well, will be useful to routinely classify patients with major psychiatric disorders, and schizophrenia in particular, on the basis of resting state functional MRI data. This technique can be a valid, cheap, and non-invasive support for physicians to detect patients, even in the early stage of the disorder, conferring a crucial diagnostic anticipation, hopefully decisive in changing the natural history of the disease. The results collected in this review allow us to assume that the greater accuracy demonstrated by the SVM models and new integrated methods of ML techniques could play an increasingly decisive role in the future both for the early diagnosis and a more accurate evaluation of the treatment response, and to establish the middle-term prognosis of patients with SCZ.

\section{AUTHOR CONTRIBUTIONS}

All authors contributed to the article and approved the submitted version.

\section{FUNDING}

This research was co-funded by University "G. Fortunato" Benevento (Italy). Grant: cda Nº/111119. 


\section{REFERENCES}

1. Owen MJ, Sawa A, Mortensen PB. Schizophrenia. Lancet (2016) 388:86-97. doi: 10.1016/S0140-6736(15)01121-6

2. McGrath J, Saha S, Chant D, Welham J. Schizophrenia: a concise overview of incidence, prevalence, and mortality. Epidemiol Rev (2008) 30:67-76. doi: 10.1093/epirev/mxn001

3. Simeone JC, Ward AJ, Rotella P, Collins J, Windisch R. An evaluation of variation in published estimates of schizophrenia prevalence from 1990-2013: A systematic literature review. BMC Psychiatry (2015) 15:193. doi: 10.1186/ s12888-015-0578-7

4. American Psychiatric Association. Diagnostic and Statistical Manual of Mental Disorders: DSM-5. fifth Ed. Washington (2013).

5. World Health Organization. The ICD-10 Classification of Mental and Behavioural Disorders. In: Clinical descriptions and diagnostic guidelines (1992). Available from: http://www.who.int/classifications/icd/en/bluebook. pdf. Accessed November 02, 2019.

6. Kambeitz J, Kambeitz-Ilankovic L, Leucht S, Wood S, Davatzikos C, Malchow B, et al. Detecting neuroimaging biomarkers for schizophrenia: A meta-analysis of multivariate pattern recognition studies. Neuropsychopharmacology (2015) 40:1742-51. doi: 10.1038/npp.2015.22

7. Senthil G, Lehner T. Schizophrenia research in the era of Team Science and big data. Schizophr Res (2019) 217:13-16. doi: 10.1016/j.schres.2019.07.008

8. He Y, Chen ZJ, Evans AC. Small-world anatomical networks in the human brain revealed by cortical thickness from MRI. Cereb Cortex (2007) 17:240719. doi: 10.1093/cercor/bhl149

9. He Y, Wang J, Wang L, Chen ZJ, Yan C, Yang H, et al. Uncovering intrinsic modular organization of spontaneous brain activity in humans. PloS One (2009) 4(4):e5226. doi: 10.1371/journal.pone.0005226

10. Han W, Sorg C, Zheng C, Yang Q, Zhang X, Ternblom A, et al. Low-rank network signatures in the triple network separate schizophrenia and major depressive disorder. NeuroImage Clin (2019) 22:101725. doi: 10.1016/ j.nicl.2019.101725

11. Veronese E, Castellani U, Peruzzo D, Bellani M, Brambilla P. Machine learning approaches: from theory to application in schizophrenia. Comput Math Methods Med (2013) 2013:867924. doi: 10.1155/2013/867924

12. Wolfers T, Buitelaar JK, Beckmann CF, Franke B, Marquand AF. From estimating activation locality to predicting disorder: A review of pattern recognition for neuroimaging-based psychiatric diagnostics. Neurosci Biobehav Rev (2015) 57:328-49. doi: 10.1016/j.neubiorev.2015.08.001

13. Orrù G, Pettersson-Yeo W, Marquand AF, Sartori G, Mechelli A. Using Support Vector Machine to identify imaging biomarkers of neurological and psychiatric disease: A critical review. Neurosci Biobehav Rev (2012) 36:114052. doi: 10.1016/j.neubiorev.2012.01.004

14. de Filippis R, Carbone EA, Gaetano R, Bruni A, Pugliese V, Segura-Garcia C, et al. Machine learning techniques in a structural and functional MRI diagnostic approach in schizophrenia: a systematic review. Neuropsychiatr Dis Treat (2019) 15:1605-27. doi: 10.2147/NDT.S202418

15. Vapnik VN. An overview of statistical learning theory. IEEE Trans Neural Networks (1999) 10:988-99. doi: 10.1109/72.788640

16. Krystal JH, Murray JD, Chekroud AM, Corlett PR, Yang G, Wang X-J, et al. Computational Psychiatry and the Challenge of Schizophrenia. Schizophr Bull (2017) 43:473-5. doi: 10.1093/schbul/sbx025

17. Deo R. Machine Learning in Medicine. Circulation (2015) 132(20):1920-30. doi: 10.1161/CIRCULATIONAHA.115.001593

18. Liberati A, Altman DG, Tetzlaff J, Mulrow C, Gøtzsche PC, Ioannidis JPA, et al. The PRISMA statement for reporting systematic reviews and metaanalyses of studies that evaluate healthcare interventions: explanation and elaboration. BMJ (2009) 339:b2700. doi: 10.1136/bmj.b2700

19. Jadad AR, Moore RA, Carroll D, Jenkinson C, Reynolds DJ, Gavaghan DJ, et al. Assessing the quality of reports of randomized clinical trials: is blinding necessary? Control Clin Trials (1996) 17:1-12. doi: 10.1016/0197-2456(95) 00134-4

20. Su L, Wang L, Shen H, Feng G, Hu D. Discriminative analysis of non-linear brain connectivity in schizophrenia: An fMRI Study. Front Hum Neurosci (2013) 7:702. doi: 10.3389/fnhum.2013.00702

21. Yang H, Liu J, Sui J, Pearlson G, Calhoun VD. A hybrid machine learning method for fusing fMRI and genetic data: Combining both improves classification of schizophrenia. Front Hum Neurosci (2010) 4:192. doi: $10.3389 /$ fnhum.2010.00192

22. Arbabshirani MR, Castro E, Calhoun VD. Accurate Classification of Schizophrenia Patients Based on Novel Resting-State fMRI Features. Conf Proc IEEE Eng Med Biol Soc (2014) 2014:6691-4. doi: 10.1109/ EMBC.2014.6945163

23. Watanabe T, Kessler D, Scott C, Angstadt M, Sripada C. Disease prediction based on functional connectomes using a scalable and spatially-informed support vector machine. Neuroimage (2014) 96:183-202. doi: 10.1016/ j.neuroimage.2014.03.067

24. Koch SP, Hägele C, Haynes JD, Heinz A, Schlagenhauf F, Sterzer P. Diagnostic classification of schizophrenia patients on the basis of regional reward-related fMRI signal patterns. PloS One (2015) 10(3):e0119089. doi: 10.1371/ journal.pone.0119089

25. Chyzhyk D, Savio A, Graña M. Computer aided diagnosis of schizophrenia on resting state fMRI data by ensembles of ELM. Neural Networks (2015) 68:2333. doi: 10.1016/j.neunet.2015.04.002

26. Liu Y, Zhang Y, Lv L, Wu R, Zhao J, Guo W. Abnormal neural activity as a potential biomarker for drug-naive first-episode adolescent-onset schizophrenia with coherence regional homogeneity and support vector machine analyses. Schizophr Res (2018) 192:408-15. doi: 10.1016/ j.schres.2017.04.028

27. Guo W, Liu F, Chen J, Wu R, Li L, Zhang Z, et al. Using short-range and longrange functional connectivity to identify schizophrenia with a family-based case-control design. Psychiatry Res - Neuroimaging (2017) 264:60-7. doi: 10.1016/j.pscychresns.2017.04.010

28. Orban P, Dansereau C, Desbois L, Mongeau-Pérusse V, Giguère CÉ, Nguyen H, et al. Multisite generalizability of schizophrenia diagnosis classification based on functional brain connectivity. Schizophr Res (2018) 192:167-71. doi: 10.1016/j.schres.2017.05.027

29. Wang S, Zhang Y, Lv L, Wu R, Fan X, Zhao J, et al. Abnormal regional homogeneity as a potential imaging biomarker for adolescent-onset schizophrenia: A resting-state fMRI study and support vector machine analysis. Schizophr Res (2018) 192:179-84. doi: 10.1016/j.schres.2017.05.038

30. Wang S, Zhan Y, Zhang Y, Lyu L, Lyu H, Wang G, et al. Abnormal long- and short-range functional connectivity in adolescent-onset schizophrenia patients: A resting-state fMRI study. Prog Neuropsychopharmacol Biol Psychiatry (2018) 81:445-51. doi: 10.1016/j.pnpbp.2017.08.012

31. Bae Y, Kumarasamy K, Ali IM, Korfiatis P, Akkus Z, Erickson BJ. Differences Between Schizophrenic and Normal Subjects Using Network Properties from fMRI. J Digit Imaging (2018) 31:252-61. doi: 10.1007/s10278-017-0020-4

32. Qureshi MNI, Oh J, Cho D, Jo HJ, Lee B. Multimodal discrimination of schizophrenia using hybrid weighted feature concatenation of brain functional connectivity and anatomical features with an extreme learning machine. Front Neuroinform (2017) 11:59. doi: 10.3389/fninf.2017.00059

33. Pläschke RN, Cieslik EC, Müller VI, Hoffstaedter F, Plachti A, Varikuti DP, et al. On the integrity of functional brain networks in schizophrenia, Parkinson's disease, and advanced age: Evidence from connectivity-based single-subject classification. Hum Brain Mapp (2017) 38:5845-58. doi: 10.1002/hbm.23763

34. Liu Y, Guo W, Zhang Y, Lv L, Hu F, Wu R, et al. Decreased Resting-State Interhemispheric Functional Connectivity Correlated with Neurocognitive Deficits in Drug-Naive First-Episode Adolescent-Onset Schizophrenia. Int J Neuropsychopharmacol (2018) 21:33-41. doi: 10.1093/ijnp/pyx095

35. Vacca A, Longo R, Mencar C. Identification and evaluation of cognitive deficits in schizophrenia using "Machine learning". Psychiatr Danub (2019) 31:261-4.

36. Zhuang $\mathrm{H}$, Liu $\mathrm{R}$, Wu C, Meng Z, Wang D, Liu D, et al. Multimodal classification of drug-naïve first-episode schizophrenia combining anatomical, diffusion and resting state functional resonance imaging. Neurosci Lett (2019) 705:87-93. doi: 10.1016/j.neulet.2019.04.039

37. Li J, Sun Y, Huang Y, Bezerianos A, Yu R. Machine learning technique reveals intrinsic characteristics of schizophrenia: an alternative method. Brain Imaging Behav (2019) 13:1386-96. doi: 10.1007/s11682-0189947-4

38. Jing R, Li P, Ding Z, Lin X, Zhao R, Shi L, et al. Machine learning identifies unaffected first-degree relatives with functional network patterns and cognitive impairment similar to those of schizophrenia patients. Hum Brain Mapp (2019) 40:3930-9. doi: 10.1002/hb 
39. Ramkiran S, Sharma A, Rao NP. Resting-state anticorrelated networks in Schizophrenia. Psychiatry Res - Neuroimaging (2019) 284:1-8. doi: 10.1016/ j.pscychresns.2018.12.013

40. Ji L, Meda SA, Tamminga CA, Clementz BA, Keshavan MS, Sweeney JA, et al. Characterizing functional regional homogeneity (ReHo) as a B-SNIP psychosis biomarker using traditional and machine learning approaches. Schizophr Res (2019) 215:430-8. doi: 10.1016/j.schres.2019.07.015

41. Zhu F, Liu Y, Liu F, Yang R, Li H, Chen J, et al. Functional asymmetry of thalamocortical networks in subjects at ultra-high risk for psychosis and firstepisode schizophrenia. Eur Neuropsychopharmacol (2019) 29:519-28. doi: 10.1016/j.euroneuro.2019.02.006

42. Zhou Y, Zeidman P, Wu S, Razi A, Chen C, Yang L, et al. Altered intrinsic and extrinsic connectivity in schizophrenia. NeuroImage Clin (2018) 17:704-16. doi: 10.1016/j.nicl.2017.12.006

43. Li P, Fan TT, Zhao RJ, Han Y, Shi L, Sun HQ, et al. Altered Brain Network Connectivity as a Potential Endophenotype of Schizophrenia. Sci Rep (2017) 7 (1):5483. doi: 10.1038/s41598-017-05774-3

44. Bisenius S, Mueller K, Diehl-Schmid J, Fassbender K, Grimmer T, Jessen F, et al. Predicting primary progressive aphasias with support vector machine approaches in structural MRI data. NeuroImage Clin (2017) 14:334-43. doi: $10.1016 /$ j.nicl.2017.02.003

45. Pellegrini E, Ballerini L, Hernandez M del CV, Chappell FM, González-Castro V, Anblagan D, et al. Machine learning of neuroimaging for assisted diagnosis of cognitive impairment and dementia: A systematic review. Alzheimer's Dement Diagnosis Assess Dis Monit (2018) 10:519-35. doi: 10.1016/j.dadm. 2018.07.004

Conflict of Interest: The authors declare that the research was conducted in the absence of any commercial or financial relationships that could be construed as a potential conflict of interest.

Copyright (c) 2020 Steardo, Carbone, de Filippis, Pisanu, Segura-Garcia, Squassina, De Fazio and Steardo. This is an open-access article distributed under the terms of the Creative Commons Attribution License (CC BY). The use, distribution or reproduction in other forums is permitted, provided the original author(s) and the copyright owner(s) are credited and that the original publication in this journal is cited, in accordance with accepted academic practice. No use, distribution or reproduction is permitted which does not comply with these terms. 